Doi: $10.32481 / \mathrm{djph} .2021 .09 .005$

\title{
On the Frontlines of the Alzheimer's Crisis:
}

\section{Advocacy Organizations in Delaware and Nationwide Urge Public Health Intervention to Curb Staggering Disease Trends}

Katie Macklin, MPA

Senior Director, Public Policy, Alzheimer's Association Delaware Valley Chapter

\begin{abstract}
Historically, Alzheimer's has incorrectly been seen as an aging issue, but more and more people are beginning to recognize that Alzheimer's is a true public health crisis. The burden is large, the impact is major, and there are clear ways that public health can intervene. Advocacy organizations, like the Alzheimer's Association, are driving changes in policy, systems and environments that address Alzheimer's and other dementia with public health solutions. In Delaware, addressing this mounting health crisis begins with employing some of the most common and effective public health tools and techniques. Through strategic action, Alzheimer's can be the next public health success story.
\end{abstract}

\section{Our "Other Healthcare Threat"}

The COVID-19 pandemic demonstrated the global impact health care crises can have on economies, health care systems, communities and individuals. Alzheimer's disease is a public health issue requiring bold action. As the number of Americans living with dementia increases each year, robust public health responses are even more critical to support those currently affected and to mitigate future impacts.

An estimated 6.2 million Americans aged 65 and older are living with Alzheimer's dementia in 2020. ${ }^{1}$ One in nine people aged 65 and older (11.3\%) has Alzheimer's dementia. ${ }^{1}$ Almost twothirds of Americans with Alzheimer's are women. ${ }^{1}$ The disease also disproportionately impacts communities of color-Black Americans are about twice as likely to have Alzheimer's or other dementias and older Hispanics are about one and one-half times as likely to have Alzheimer's or other dementias as compared to older Whites. ${ }^{1}$ Furthermore, as the number of older Americans grows rapidly, so too will the number of new and existing cases of Alzheimer's. By 2050, the number of people aged 65 and older with Alzheimer's dementia is projected to reach 12.7 million. ${ }^{1}$

In Delaware, the prevalence and incidence of the disease are equally as daunting. It was estimated that in 2020, 19,000 Delawareans aged 65 and older were living with Alzheimer's. ${ }^{1}$ And this growth is expected to continue - between 2020 and 2025, the number of Delawareans aged 65 and over living with Alzheimer's is projected to increase by more than $21 \%{ }^{1}$

Alzheimer's is one of the country's most expensive diseases. In 2021, Alzheimer's and other dementia is estimated to cost the nation $\$ 355$ billion, including \$239 billion in Medicare and Medicaid payments. ${ }^{1}$ This number is projected to reach more than $\$ 1.1$ trillion by 2050 (in 2021 dollars). ${ }^{1}$ This dramatic rise includes more than three-fold increases both in government spending under Medicare and Medicaid and in out-of-pocket spending for individuals and families. In Delaware, the estimated Medicaid costs of caring for Delawareans with Alzheimer's was $\$ 253$ million $^{1}$ in 2020 . By 2025 , these costs are projected to increase by more than $23 \%{ }^{1}$ 
The impact of Alzheimer's and other dementias on the nation's health care system is also becoming catastrophic. Staggeringly, more than one quarter of all hospitalizations of people with dementia are preventable. Ninety-five percent of people living with Alzheimer's and other dementias have at least one other chronic condition - such as heart disease, diabetes, or strokeand the very nature of having Alzheimer's complicates the management of these other chronic conditions. ${ }^{1}$ This can result in poorer health outcomes and increase health care costs-both for the individual and the health care system.

In addition to the individual living with the disease, Alzheimer's impacts a network of others who provide care and support. In 2020 alone, there were an estimated 46,000 Delaware caregivers who provided $67,000,000$ total hours of unpaid care valued at $\$ 1,141,000,000$. Nationwide last year, more than 11 million Americans provided over 18 billion hours of unpaid care for people living with Alzheimer's. This results in an additional $\$ 10$ billion in increased health care costs due to the burden of caregiving. ${ }^{1}$

But the costs of Alzheimer's and other dementias are more than financial. This is why the Alzheimer's Association unrelentingly advocates for the rights of the Alzheimer's and dementia community, as well as critical research funding. Enhancing care and support for all those impacted by the disease today will change the trajectory of this disease for future generations. In order to truly make a real impact - each and every day - in the lives of those living with Alzheimer's and their caregivers, it is critical that grassroots advocacy and change take place at the state and local level-here in Delaware. State and local health agencies and community partners are key players in this arena.

\section{Public Health and the Fight Against Alzheimer's}

Public health works to improve the health and well-being of an entire population - at all stages of life. We know that changes in the brain associated with Alzheimer's begin years, even decades, before symptoms appear. Certain risk factors - such as smoking, physical inactivity, and heart disease - may contribute to Alzheimer's dementia, but are modifiable. That is why a life-course approach to Alzheimer's and other dementias is imperative, and why public health has numerous opportunities to intervene across the lifespan. Such public health opportunities include:

- Risk Reduction: In the public health realm, an essential service is preventing disease, disability, and premature death among populations. At any stage of life, public health can reduce the risk of cognitive decline by promoting cognitive health and healthy behaviors - such as quitting smoking, protecting the head from brain injuries, being physically active, and eating a nutritious diet.

- Early Detection: Public health can promote early detection and diagnosis by ensuring that the general public understand the early warning signs of cognitive decline and that health care providers - of all specialties - are informed, educated, and stay up-to-date with the latest cognitive health science.

- Safety and Quality of Care: For people living with dementia, public health can enhance the quality of care through provider education, and can educate caregivers about available services and supports in the community. 
Advocacy organizations like the Alzheimer's Association and Alzheimer's Impact Movement (AIM), in coordination with volunteer advocates, play a critical role in efforts to influence Alzheimer's and dementia policy at the federal and state level. Through advocacy, these organizations help bring attention to and encourage policymakers to take action. In order for Delaware to be a more dementia capable state, Delaware public health officials must work to educate health care providers and the public about the importance of early detection and diagnosis, promote cognitive health and risk reduction across all ages, and improve access to and awareness of resources.

To identify specific ways to take action, officials should look to the Healthy Brain Initiative (HBI), a public-private initiative that aims to advance cognitive health as a central component of public health practice. ${ }^{2}$ The HBI State and Local Public Health Partnerships to Address Dementia: The 2018-2023 Road Map, or the HBI Road Map, offers 25 actions that public health agencies, organizations, and partners can take to address dementia using a population health approach. ${ }^{3}$ The HBI Road Map supports core functions of public health and is aligned to objectives of Healthy People 2030 and the Essential Public Health Services. ${ }^{4}$ The following demonstrate how Delaware can shape its own response to Alzheimer's and dementia, and how advocacy organizations can incorporate public health action into their policy goals and advocacy agenda:

- Educate and Empower Communities: Seven actions of the HBI Road Map have this specific aim. This includes calling on public health agencies to educate the general public about cognitive health and dementia, changes in cognition that they should discuss with a health professional, and the benefits of obtaining an early diagnosis.

- Develop Policies and Mobilize Partnerships: Creating plans, programming, and initiatives at the policy or systems level related to cognitive decline, Alzheimer's, and caregiving can expand reach and result in longer-lasting impacts as compared to individual interventions. Six HBI Road Map actions are focused on developing policies and mobilizing partnerships. For example, public health agencies could partner with community-based and private organizations to establish policies that promote supportive communities and workplaces for dementia caregivers.

Population-wide policies that support and maintain the health, well-being, and productivity of caregivers could be developed through convening key partners and caregivers to use data and evidence to recognize issues and gaps, and create solutions to address them.

- Assure a Competent Workforce: Both public health professionals and the health care workforce must know how to accurately, appropriately, and comprehensively assess cognitive impairment while applying principles of person-centered care. Seven actions of the HBI Road Map aim to help the public health community ensure a competent workforce, including educating public health professionals about the best available evidence on dementia and caregiving; the role of public health in addressing cognitive issues; and sources of information, tools, and assistance to support action.

- Monitor and Evaluate: Data about the prevalence, scope, and burden of Alzheimer's, dementia, cognitive issues, and caregiving are used to shape 
priorities, allocate resources, identify gaps, and address disparities. Healthy People $2030^{4}$ tracks awareness of dementia, hospitalizations in older adults with dementia, and older adults with subjective cognitive decline who have discussed symptoms with a health care provider. The HBI Road Map includes five actions aimed at improving monitoring and evaluation to ensure the greatest possible impact and change. One such action calls on public health agencies and partners to use data to inform the program and policy responses to cognitive health, impairment, and caregiving. To assist public health agencies on this front, the Behavioral Risk Factor Surveillance System (BRFSS) offers two modules - the Cognitive Decline Module and the Caregiver Module - that collect populationbased data on cognitive decline and caregiving issues. In fact, the Cognitive Decline Module serves as the data source for Healthy People 2030 Objective DIA-03, which aims to increase the proportion of adults experiencing subjective cognitive decline who discuss their symptoms with a health care provider. The Alzheimer's Association and the Alzheimer's Disease and Healthy Aging Program at the Centers for Disease Control and Prevention (CDC) both produce national, state-specific, and population-specific infographics and facts sheets with this data for use by public health agencies and partners. Additionally, the Alzheimer's Disease and Healthy Aging Data Portal provides easy access to national- and state-level data on a range of key indicators of health and well-being for older adults, including cognitive health and caregiving. ${ }^{5}$

\section{Much Has Been Achieved, More Work Remains}

Through public policy, the State of Delaware has the opportunity to reach the masses of people impacted by dementia today, and change the trajectory of this disease for future generations. Through advocacy, Alzheimer's can become a true national and state priority, and remain front of mind for policymakers. Recent public policy successes will contribute to state and federal efforts to address dementia as a public health issue, using public health tools and approaches. These are just examples, to illustrate the foundation that has been laid and the ongoing need for more action:

- The Building Our Largest Dementia (BOLD) Infrastructure for Alzheimer's Act (PL 115-406), enacted into federal law in 2018, creates an Alzheimer's public health infrastructure across the country and increases implementation of the HBI Road Map by state, local, and tribal public health agencies. This legislature provides for the establishment of Alzheimer's centers of excellence, cooperative agreements to public health departments to implement the HBI Road Map, and expanded public health data collection. The awarded public health departments are operationalizing the HBI Road Map, implementing actions to reach the most people, for the most impact.

- In 2010, the Alzheimer's Association and AIM, worked with Congress to develop the National Alzheimer's Project Act (NAPA). Signed into law in 2011, NAPA led to the creation of a national strategic plan to address the rapidly escalating Alzheimer's crisis and fundamentally changed the way the nation addresses Alzheimer's and all other dementia. ${ }^{6}$ 
- The bipartisan Promoting Alzheimer's Awareness to Prevent Elder Abuse Act (S.3703/H.R. 6813), legislation championed by the Alzheimer's Association and the AIM, was signed into law in 2020. This new law requires the Department of Justice (DOJ) to develop materials designed to assist law enforcement officers, prosecutors, judges, medical personnel, victims' services personnel and others who encounter and support individuals living with Alzheimer's and other dementia.

- Also signed into law in 2020, the Improving HOPE for Alzheimer's Act (S. 880/H.R. 1873), requires the Centers for Medicare \& Medicaid Services (CMS) to conduct education and outreach about care planning services available for individuals living with Alzheimer's or another dementia to care providers. The law also requires a report to Congress on provider outreach and utilization rates, including information on any barriers Medicare beneficiaries face in accessing these services and recommendations to address those barriers.

- Signed into law in 2018, the RAISE Family Caregivers Act addresses the needs of our nation's family caregivers through the development and implementation of a coordinated national strategy.

- Understanding the devastating toll Alzheimer's takes on American families and the economy, increasing government investment in research has become a bipartisan priority in Congress, and federal appropriations for Alzheimer's and dementia research funding at the National Institutes of Health (NIH) has increased more than seven-fold since 2011. Annual federal investment in Alzheimer's and dementia research is now at $\$ 3.1$ billion.

These federal public policy successes specific to the Alzheimer's and dementia community will lead to direct impacts for Delawareans. In 2012, Delaware embarked on the development of its own comprehensive state plan, outlining strategic goals and objectives toward becoming a more dementia capable state. The Delaware State Plan to Address Alzheimer's Disease and Related Disorders was published in 2013 following an inclusive and transparent process involving key stakeholders and the public. The plan outlines actions to: increase awareness of and understanding about Alzheimer's disease; bring focused attention to the development of longterm care services for persons with Alzheimer's disease; strengthen support for caregivers; improve the capacity of Delaware's workforce to respond to the needs of persons with Alzheimer's disease; and increase Delaware's capacity for Alzheimer's disease-related research and data collection. These specific areas all align with the core components of utilizing a public health approach.

Delaware is taking steps towards becoming a more dementia capable state and in addressing Alzheimer's from a public health lens. This includes a variety of programs to support caregivers, an online Alzheimer's Toolkit linked to from the Department of Health and Social Services (DHSS) webpage; the Division of Services for Aging and Adults with Physical Disabilities (DSAAPD) working in collaboration with the Alzheimer's Association on a public awareness campaign to promote early detection and diagnosis, to be launched next year; and a number of bills pending in the legislature that will provide more support for caregivers, enhance home and community based services and address healthcare workforce development. 
Doi: $10.32481 /$ djph.2021.09.005

\section{Conclusion}

The Alzheimer's crisis requires bold action. There is a tremendous amount of dementia specific work to accomplish at both the federal and state levels. It is imperative to work across sectors to better understand the public health burden of cognitive impairment through surveillance; building a strong base of evidence for policies, communication, and programmatic interventions for promoting cognitive health and addressing cognitive impairment and caregiving; and translating that foundation into effective public health practices throughout Delaware and across the country. Educating elected officials on the impact of Alzheimer's and other dementias is essential to increasing public awareness and concern. It is imperative that policymakers, advocacy organizations, healthcare systems, community-based organizations and providers, and many, many others work collaboratively to ensure the issues facing the Alzheimer's and dementia community remain at the forefront. It is through this collaboration and the collective power of those across Delaware, that real, lasting change can occur-improving the lives of those with Alzheimer's and their caregivers and improving the systems that are in place to serve these communities.

The federal government must continue its strong commitment to funding Alzheimer's and dementia research at the National Institutes of Health (NIH), as well as fully funding the implementation of the BOLD Infrastructure for Alzheimer's Act at the Centers for Disease Control and Prevention (CDC) each year. Funding for NIH research will help lead to new treatments, prevention and ultimately a cure; and BOLD funding will enable CDC to expand the number of state, local and tribal public health departments that receive funding for Alzheimer's public health action.

At the state level, Delaware policymakers and public health officials must also take action. For example: addressing the risk factors for dementia among vulnerable populations; advancing early detection and diagnosis of mild cognitive impairment and dementia; improving safety and quality of care for people living with dementia; and providing better support for caregivers.

With so many Delawareans impacted by this public health crisis, the time to act is now. Our state and federal legislators need to understand the scope and the scale of the Alzheimer's crisis - it is key to moving impactful policy.

\section{References}

1. Alzheimer's Association. (2021). 2021 Alzheimer's Disease Facts and Figures. Retrieved from https://www.alz.org/media/Documents/alzheimers-facts-and-figures.pdf

2. Centers for Disease Control and Prevention. (2020, Jul 30). Healthy Brain Initiative. Retrieved from https://www.cdc.gov/aging/healthybrain/index.htm

3. Alzheimer's Association. (2018). Healthy Brain Initiative: State and Local Public Health Partnerships to Address Dementia: The 2018-2023 Road Map. Healthy Brain Initiative. Retrieved from https://www.alz.org/media/Documents/healthy-brain-initiative-road-map2018-2023.pdf

4. U.S. Department of Health and Human Services. (n.d.). Healthy People 2030. Retrieved from https://health.gov/healthypeople 
Doi: $10.32481 /$ djph.2021.09.005

5. Centers for Disease Control and Prevention. (2019, Sep 9). Alzheimer's Disease and Healthy Aging Data Portal. Retrieved from https://www.cdc.gov/aging/agingdata/index.html

6. U.S. Department of Health and Human Services. (2021, Apr 27). National Alzheimer's Project. Retrieved from https://aspe.hhs.gov/national-alzheimers-project-act\#NAPA

Copyright (c) 2021 Delaware Academy of Medicine / Delaware Public Health Association.

This is an Open Access article distributed under the terms of the Creative Commons Attribution Non-Commercial License (https://creativecommons.org/licenses/by-nc-nd/4.0/) which permits unrestricted non-commercial use, distribution, and reproduction in any medium, provided the original work is properly cited. 